\title{
Coherent Magnetic Response at Optical Frequencies Using Atomic Transitions
}

\author{
Nicholas R. Brewer, Zachary N. Buckholtz, Zachary J. Simmons, Eli A. Mueller, and Deniz D. Yavuz \\ Department of Physics, 1150 University Avenue, University of Wisconsin at Madison, \\ Madison, Wisconsin 53706, USA
}

(Received 24 October 2016; revised manuscript received 9 December 2016; published 23 January 2017)

\begin{abstract}
In optics, the interaction of atoms with the magnetic field of light is almost always ignored since its strength is many orders of magnitude weaker compared to the interaction with the electric field. In this article, by using a magnetic-dipole transition within the $4 f$ shell of europium ions, we show a strong interaction between a green laser and an ensemble of atomic ions. The electrons move coherently between the ground and excited ionic levels (Rabi flopping) by interacting with the magnetic field of the laser. By measuring the Rabi flopping frequency as the laser intensity is varied, we report the first direct measurement of a magnetic-dipole matrix element in the optical region of the spectrum. Using density-matrix simulations of the ensemble, we infer the generation of coherent magnetization with magnitude $5.5 \times 10^{-3} \mathrm{~A} / \mathrm{m}$, which is capable of generating left-handed electromagnetic waves of intensity $1 \mathrm{nW} / \mathrm{cm}^{2}$. These results open up the prospect of constructing left-handed materials using sharp transitions of atoms.
\end{abstract}

DOI: 10.1103/PhysRevX.7.011005

\section{INTRODUCTION}

Optics and optical tools have played a key role in the development of the physical sciences and engineering over the past century. In particular, after the invention of the laser in 1960, optical techniques have penetrated into and revolutionized many fields of science including biological imaging and nanotechnology. Over the past two decades, a new generation of optical tools have been invented and continually refined, using materials that are not available in nature [1-6]. These materials, termed metamaterials, offer the promise of devices with unique capabilities: for example, lenses that can image objects with a resolution much better than the wavelength of the imaging light $[7,8]$, and the ability to cloak objects for certain regions of the spectrum [9-11]. The unique capabilities of metamaterials result from their unusual optical properties such as having a negative index of refraction and, consequently, the lefthanded nature of the propagating electromagnetic waves. It is now well understood that metamaterials rely critically on their ability to strongly interact with both the electric and the magnetic fields of light; i.e., both the permittivity and permeability of the material must be substantially different from their values in free space. This is a big challenge in the optical region of the spectrum since materials generally do not respond to the magnetic field of light at such high

Published by the American Physical Society under the terms of the Creative Commons Attribution 4.0 International license. Further distribution of this work must maintain attribution to the author(s) and the published article's title, journal citation, and DOI.
Subject Areas: Atomic and Molecular Physics, Optics, Photonics

frequencies. As demonstrated by the experiments from the metamaterial community, this challenge can be overcome using devices that are engineered at the nanoscale. These devices utilize features such as small metallic loops that couple resonantly to light at specific wavelengths and produce magnetic dipoles oscillating at high frequencies [12]. Because of their metallic nature, such devices usually exhibit large losses, and much work over the past decade has focused on overcoming losses and constructing metamaterials with low absorption [13-15].

It was recognized early on that it may also be possible to construct left-handed materials using sharp transitions of atoms or ions [16-20]. When an electron makes a transition from the ground level to an excited level, it can do so by either interacting with the electric field or the magnetic field of light. Depending on the nature of the interaction, these transitions are said to be electric dipole or magnetic dipole. A naturally occurring left-handed material would require both types of transitions simultaneously. The chief difficulty of this approach is that in the optical region of the spectrum one essentially encounters only electric-dipole transitions since they are stronger by about 5 orders of magnitude. Near such electric-dipole transitions the permittivity of the material can be strongly modified, giving rise to a whole family of optical effects. For example, using electromagnetically induced transparency and related ideas, light can be slowed down, stopped, or even forced to move backward, and optical nonlinearities can be made large enough to be effective at the single-photon level [21-28]. Although these are exciting developments, it is important to note that these atomic physics experiments are not truly comparable to metamaterials work since there is negligible interaction with the magnetic field of light. 
In this article, we show a strong coherent interaction between the magnetic field of a laser beam and an ensemble of atomic ions. We use a cryogenically cooled europiumdoped crystal and utilize the ${ }^{7} F_{0} \rightarrow{ }^{5} D_{1}$ magnetic-dipole transition within the $4 f$ shell. By using an intense laser beam at a green wavelength of $527.5 \mathrm{~nm}$, we observe strong-field Rabi flopping between the ground and excited levels. We measure the change in the Rabi flopping frequency as the laser intensity is varied and deduce the magnetic-dipole matrix element to be $\mu=(0.063 \pm$ $0.005) \mu_{B}$ (the quantity $\mu_{B}$ is the Bohr magneton). We verify the magnetic-dipole nature of the excitation by studying angle-dependent fluorescence from the crystal. As we discuss below, our matrix element and probe absorption measurements demonstrate a magnetic susceptibility exceeding $\chi_{M}>10^{-5}$. We perform density-matrix simulations of the system and our numerical results fit well to the observed Rabi flopping data. From these simulations, we infer that during the Rabi cycle the ionic ensemble reaches a peak magnetization of $5.5 \times 10^{-3} \mathrm{~A} / \mathrm{m}$, which is capable of supporting left-handed electromagnetic waves of intensity $1 \mathrm{nW} / \mathrm{cm}^{2}$ [29]. Using laser excitation through a sharp atomic transition (with widths at the $\mathrm{GHz}$ level), our experiments show for the first time that (i) a large coherent magnetic response can be obtained from an atomic ensemble and (ii) the ensemble can have a permeability that is significantly modified compared to its free-space value. These results open up the prospect of constructing lefthanded materials using atomic transitions, a different and in many ways complementary approach to existing lefthanded metamaterials.

\section{EXPERIMENTAL SCHEMATIC}

The existence of magnetic-dipole transitions in the optical region of the spectrum has been known for quite some time [30-32]. Early spectroscopic studies of rare-earth doped crystals predicted the magnetic-dipole nature of some of the transitions by studying the polarization properties of their fluorescence $[33,34]$. The oscillator strengths of these transitions are weaker by a factor of $\alpha^{2}$ (the quantity $\alpha \approx 1 / 137$ is the fine-structure constant) compared to electric-dipole transitions. As a result, accessing these transitions is difficult since they are easily overwhelmed by nearby electric-dipole transitions or are largely broadened by dissipative processes. Laser excitation of a magnetic-dipole transition was achieved only last year in the pioneering work of Novotny and colleagues [35]. They used europium-doped nanoparticles at room temperature and conclusively demonstrated the magnetic dipole nature of the ${ }^{7} F_{0} \rightarrow{ }^{5} D_{1}$ transition by utilizing a radially polarized focused laser. To our knowledge, the only detailed spectroscopic study of the ${ }^{7} F_{0} \rightarrow{ }^{5} D_{1}$ transition was performed by Shen and Kachru about two decades ago [36].

To access the ${ }^{7} F_{0} \rightarrow{ }^{5} D_{1}$ transition in our experiment, we start with an infrared external cavity diode laser (ECDL) at a wavelength of $1055 \mathrm{~nm}$. Figure 1 shows the simplified schematic of our experiment. The ECDL is locked to an ultralow expansion (ULE) glass cavity that serves as a frequency reference. The ECDL output is then amplified to an optical power exceeding $5 \mathrm{~W}$ using a fiber amplifier. We frequency double the fiber amplifier output using cavitybased second harmonic generation with a periodically poled nonlinear crystal (PPKTP). Using this setup, we are able to produce green light at a wavelength of $527.5 \mathrm{~nm}$ with an optical power exceeding $1.5 \mathrm{~W}$ and an absolute frequency stability at the $50-\mathrm{kHz}$ level. The green output from the doubling cavity is then split into two beams. Each beam goes through an acousto-optic modulator (AOM) for frequency and timing control. We use a 3-mm-long europium-doped yttrium orthosilicate crystal $\left(\mathrm{Eu}^{+3}: \mathrm{Y}_{2} \mathrm{SiO}_{5}\right.$, hereafter abbreviated as Eu:YSO), which is housed in a continuous-flow liquid helium cryostat and cooled to a temperature of $4.5 \mathrm{~K}$. We were motivated to use this

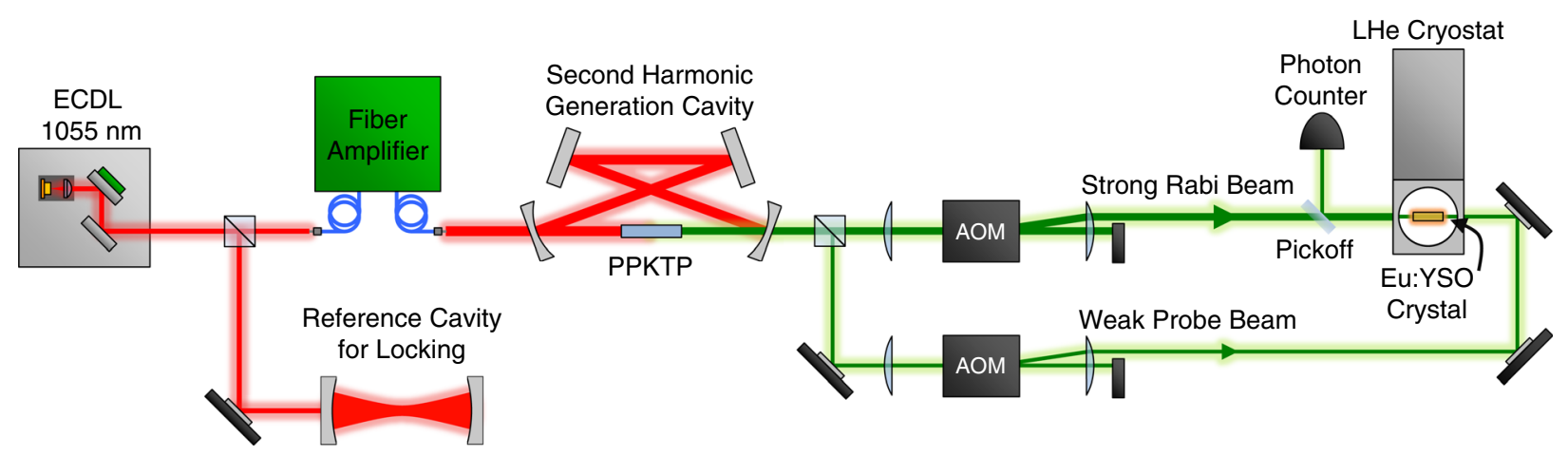

FIG. 1. The simplified experimental schematic. The experiment starts with an infrared external cavity diode laser (ECDL) at a wavelength of $1055 \mathrm{~nm}$. The ECDL is locked to a reference cavity which is constructed from ultralow expansion glass. The fiber amplifier produces an optical power exceeding $5 \mathrm{~W}$ while maintaining the frequency stability of the ECDL. The fiber amplifier output is frequency doubled using cavity-based second harmonic generation with a periodically poled KTP (PPKTP) crystal. The output of the second harmonic generation cavity is split into two beams and acousto-optic modulators (AOM) are used to precisely control frequency and timing. The Eu:YSO crystal is housed in a continuous-flow liquid-helium cryostat. 
specific crystal by the recent quantum storage experiments using the forced electric-dipole ${ }^{7} F_{0} \rightarrow{ }^{5} D_{0}$ transition $[37,38]$. The crystal is cut such that light propagates along the $b$ optical axis and the magnetic field of the laser is aligned with the $D_{1}$ axis. Further details regarding our experimental system can be found in Appendixes A and B.

The $4 f$ shell of rare-earth ions remains close to the ionic core and is relatively well shielded from the crystalline environment by the filled $5 s$ and $5 p$ shells. The levels of the $4 f$ shell of $\mathrm{Eu}^{+3}$ ions with an energy below $20000 \mathrm{~cm}^{-1}$ are shown in Fig. 2. Our crystal is doped at $\sim 0.1 \%$ with natural abundance europium, containing $48 \%{ }^{151} \mathrm{Eu}$ and $52 \%{ }^{153} \mathrm{Eu}$. Each of these isotopes has a nuclear spin of $I=5 / 2$. The hyperfine structure of the ${ }^{153} \mathrm{Eu}$ isotope is also displayed in Fig. 2 (the other isotope has smaller separations between the hyperfine levels). The upper plot in Fig. 2 shows the transmission of a weak green probe laser beam as its frequency is scanned through the ${ }^{7} F_{0} \rightarrow{ }^{5} D_{1}$ resonance (with the Rabi beam turned off). We observe an inhomogeneously broadened width of $1.6 \mathrm{GHz}$ with an onresonance optical depth of 1.8 through our crystal [i.e., the transmission at the line center is $\exp (-1.8)=0.17]$. The hyperfine structure is not resolved in the absorption spectrum since the spacing between the hyperfine levels is small compared to the inhomogeneous linewidth.

For Rabi flopping, the strength of the interaction between the light and atoms must be large compared to dissipative rates in the system. For magnetic-dipole transitions, the interaction strength can be summarized through the Rabi frequency, $\Omega=B \mu / \hbar$, and we require $\Omega \gg \Gamma$, where $\Gamma$ is the homogeneous linewidth of the transition. In our system, the dominant contribution to $\Gamma$ is phonon-induced relaxation of the excited level to its closest neighbor, the ${ }^{5} D_{0}$ level. The lower plot in Fig. 2 shows the measurement of the homogeneous linewidth $\Gamma$. Here, following excitation of the ions to the excited level ${ }^{5} D_{1}$ using a short laser pulse, we record the fluorescence from the ${ }^{5} D_{0}$ level to the lower $F$ manifold as a function of time. The initial sharp rise in the fluorescence is due to phonon-induced relaxation from ${ }^{5} D_{1}$ to ${ }^{5} D_{0}$, showing a nonradiative lifetime of $\tau=33 \mu \mathrm{s}$, and, therefore, a homogeneous linewidth of $\Gamma=2 \pi \times 4.8 \mathrm{kHz}$, which is consistent with the measurements of Ref. [36]. As we discuss below, we can achieve Rabi frequencies approaching $\Omega \approx 2 \pi \times 1 \mathrm{MHz}$, which is well within the strong drive regime $\Omega \gg \Gamma$. The slow decay of the fluorescence in the lower plot of Fig. 2 is due to the radiative lifetime of the ${ }^{5} D_{0}$ level.

\section{RABI FLOPPING}

We proceed with a discussion of our Rabi flopping measurements. With the ions starting in the ground state ${ }^{7} F_{0}$ (equally populating the three hyperfine levels), we apply a Rabi flopping laser pulse (optical power of
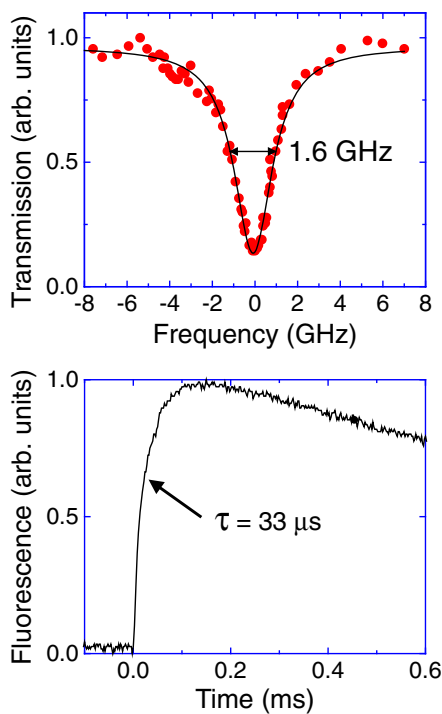

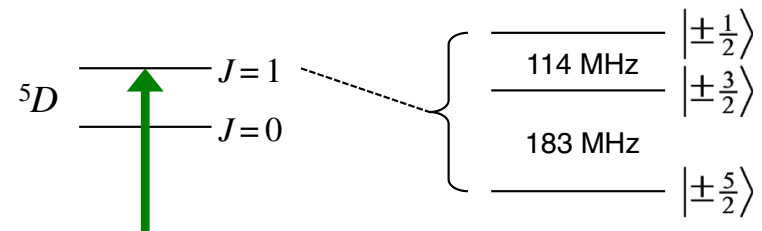

$527.5 \mathrm{~nm}$

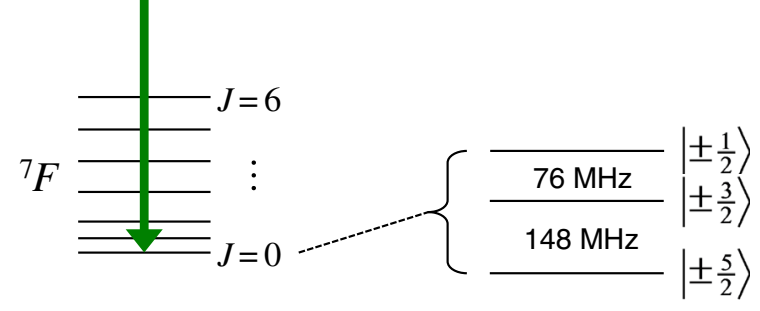

FIG. 2. The levels of the $4 f$ shell of $\mathrm{Eu}^{+3}$ ions with an energy below $20000 \mathrm{~cm}^{-1}$. We use the ${ }^{7} F_{0} \rightarrow{ }^{5} D_{1}$ magnetic-dipole transition at a wavelength of $527.5 \mathrm{~nm}$. The hyperfine structure of the ${ }^{153} \mathrm{Eu}^{+3}$ isotope with a nuclear spin of $I=5 / 2$ is also shown (the ordering of the upper hyperfine levels is not known). The numbers in the brackets are the projection of the nuclear spin angular momentum, $\left| \pm m_{I}\right\rangle$. The upper plot shows the transmission of a weak probe laser beam as its frequency is scanned across the magnetic-dipole transition. We observe an inhomogeneously broadened line (which is due to variations in the crystal field and crystal strains) with a width of $1.6 \mathrm{GHz}$, and a peak absorption of $\exp (-1.8)$. The lower plot shows the measurement of the homogeneous linewidth. Here, following excitation to the ${ }^{5} D_{1}$ level, we record the fluorescence from the ${ }^{5} D_{0}$ level to the ground $F$ manifold. The initial sharp rise in the fluorescence is due to phonon-induced relaxation from ${ }^{5} D_{1}$ to ${ }^{5} D_{0}$, which results in a homogeneous linewidth of $\Gamma=2 \pi \times 4.8 \mathrm{kHz}$ (corresponding to a nonradiative lifetime of $\tau=33 \mu \mathrm{s}$ ). The slow decay in the fluorescence is due to the radiative lifetime of the ${ }^{5} D_{0}$ level. 
$\sim 100 \mathrm{~mW}$, focused to a $1 / e^{2}$ intensity radius of $56 \mu \mathrm{m}$ ) of certain duration to the ions. Because of the interaction with the Rabi flopping laser, the ionic population starts to flow coherently back and forth between the ground and excited levels. Depending on the duration of the Rabi pulse, the ions may be left in any one of the levels or in a superposition of the two at the end of the pulse. After the Rabi flopping laser is turned off, we turn on a counterpropagating weak probe pulse (optical power less than $1 \mathrm{pW}$ ) and measure the transmission of the probe through the crystal with a photon counter (see Appendix $\mathrm{C}$ for further details on the experimental timing sequence). The spatial size of the probe at the crystal is small compared to the size of the Rabi beam so that the probe laser samples the peak of the Rabi laser spatial profile. Using this setup, the Rabi flopping of the ions is then revealed by the transmission of the probe laser beam, through stimulated absorption and emission. Figure 3 shows the normalized transmission of the probe laser through the crystal as the duration of the Rabi pulse is varied. For each pulse duration, each data
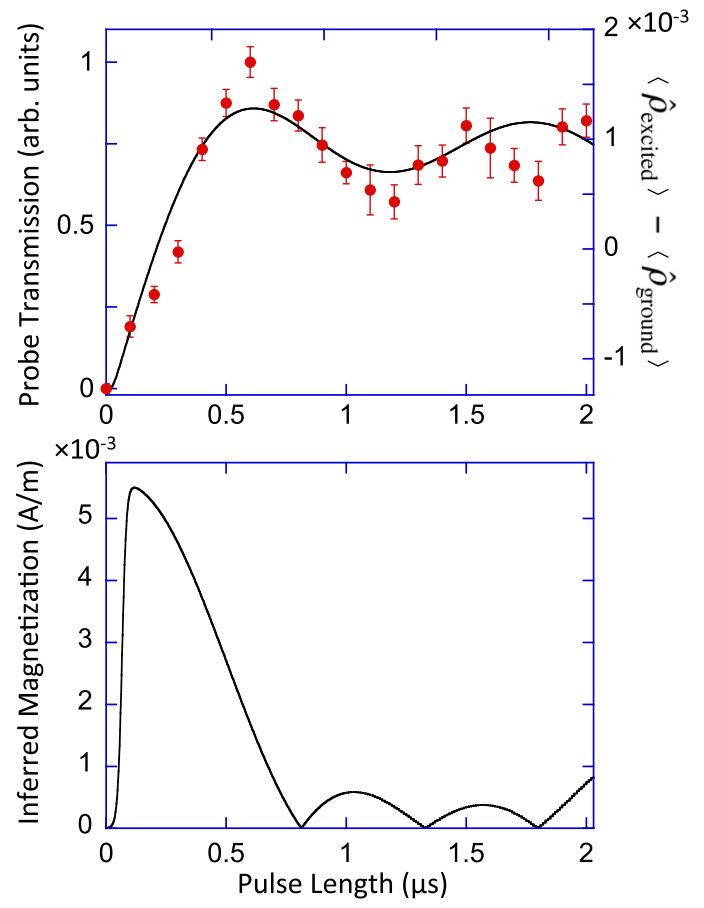

FIG. 3. The transmission of the probe laser beam through the crystal as the duration of the Rabi pulse is varied. We observe a clear Rabi cycle with a frequency approaching $1 \mathrm{MHz}$. The Rabi oscillation quickly dephases due to a number of processes, such as the variation of the magnetic-dipole matrix element among various hyperfine transitions. The solid line is a numerical simulation that solves the density matrix for a large number of atoms whose absolute transition frequencies are spread through the inhomogeneous profile, causing different hyperfine transitions to be resonant with the laser. The lower plot shows the inferred magnetization from the same simulations. See text for details. point is an average of ten experiments with the error bar denoting the standard deviation of the set. We observe a clear Rabi flopping cycle with a flopping frequency approaching $1 \mathrm{MHz}$. The Rabi flopping quickly dephases after about two cycles due to two main reasons. (i) We perform this experiment using the whole inhomogeneously broadened ensemble. As a result, the Rabi laser is only resonant with a small fraction of ions. Off-resonant atoms flop at faster rates but with a much reduced contrast, which contributes to the dephasing of the Rabi cycle. (ii) Since we do not perform any optical pumping between hyperfine levels, Rabi flopping happens simultaneously between each ground and excited hyperfine level combination; i.e., there are nine different simultaneous excitations. As we discuss in Appendix E, the spread of the magnetic-dipole matrix element between respective transitions also contributes to the fast dephasing of the Rabi cycle.

Because we observe only about two cycles, there is significant uncertainty in the observed Rabi frequency from a single flopping experiment. For accurate measurement of the magnetic dipole matrix element, we perform the Rabi flopping measurement of Fig. 3 at various laser pulse intensities. Figure 4 shows the observed Rabi frequency as a function of laser intensity for 23 Rabi flopping measurements. For each measurement, the vertical error bar represents the uncertainty of the deduced Rabi frequency for that specific flopping experiment. The right-hand panels show six specific Rabi flopping experiments as the laser intensity is varied from 5630 to $1510 \mathrm{~W} / \mathrm{cm}^{2}$. From the square-root fit to the observed data values (the solid line in the main plot), we deduce the magnetic-dipole matrix element to be $\mu=(0.063 \pm 0.005) \mu_{B}$ for the magnetic field oriented along the quantization axis of the crystal (the $D_{1}$ optical axis). It is well known that the $4 f$ electronic configuration of rare-earth ions is in many cases in the intermediate coupling regime; i.e., neither $L S$ coupling nor $j j$ coupling accurately describes the wave functions. Although highly complex, the wave functions of the $4 f$ shell of $\mathrm{Eu}^{+3}$ have been reasonably well understood since the pioneering work of Ofelt and others many decades ago [39-41]. Using intermediate-coupled wave functions and well-known methods of Racah algebra, calculations give a theoretical value of $\mu=0.096 \mu_{B}$ for the magnetic-dipole matrix element (see Appendix D for further details). The calculated theoretical value is therefore within $50 \%$ of the measured experimental value of the matrix element. The reasons for the discrepancy will be among our future investigations.

With the measured value of the magnetic-dipole matrix element, we perform density-matrix simulations of the system. For this purpose, we take 80000 ions, spread their transition frequencies across the inhomogeneous profile, numerically solve the time-domain evolution of the density matrix for each ion, and calculate the ensemble-averaged response. For the time-domain evolution, we include all six 

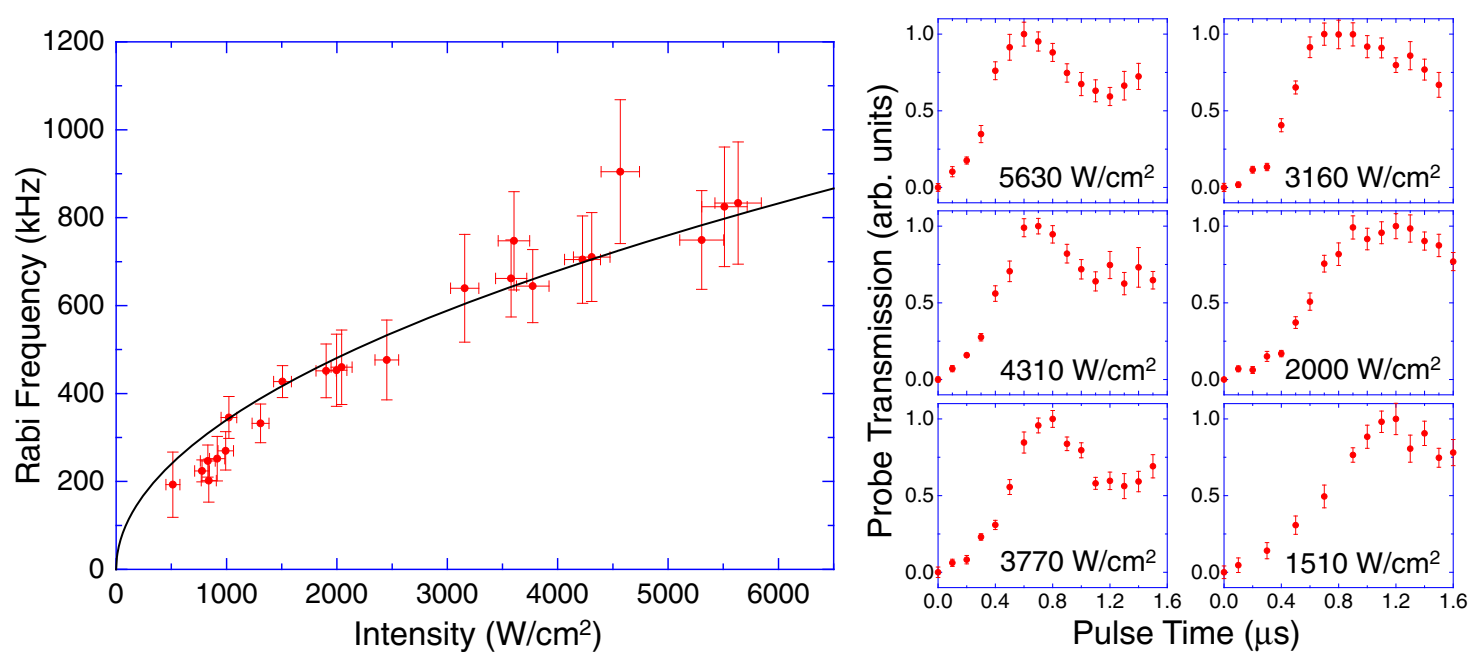

FIG. 4. The observed Rabi flopping frequency as the laser intensity is varied for 23 different Rabi flopping experiments. From the square-root fit to the data points (the solid line), we deduce a magnetic-dipole matrix element of $\mu=(0.063 \pm 0.005) \mu_{B}$. The right-hand panels show six specific flopping experiments (probe transmission versus Rabi pulse duration) as the laser intensity is varied from 5630 to $1510 \mathrm{~W} / \mathrm{cm}^{2}$.

hyperfine levels of the ${ }^{7} F_{0} \rightarrow{ }^{5} D_{1}$ transition as well as the ${ }^{5} D_{0}$ level; i.e., the size of the density matrix $\hat{\rho}$ is $7 \times 7$. Our model takes into account (i) interaction with the laser pulse, (ii) nonradiative relaxation from the ${ }^{5} D_{1}$ level to the ${ }^{5} D_{0}$ level at a rate $\Gamma$ (homogeneous linewidth), and (iii) the spread of the transition frequencies due to the inhomogeneous broadening (see Appendix E for further details on the numerical simulations). The solid line in Fig. 3 shows the calculated difference between the population of the excited hyperfine levels and the ground hyperfine levels, $\left\langle\hat{\rho}_{\text {excited }}\right\rangle-\left\langle\hat{\rho}_{\text {ground }}\right\rangle$ (we use the notation $\langle\cdots\rangle$ to mean average over the inhomogeneously broadened ensemble), as the Rabi pulse duration is varied. The numerical simulation accurately produces the frequency and the contrast observed in the Rabi flopping experiment. In the lower plot in Fig. 3, we calculate the inferred magnetization of the ensemble by extracting the coherences (the off-diagonal density matrix elements) from the same numerical simulations, $M=$ $N\left\langle\hat{\rho}_{\text {off-diagonal }}\right\rangle \mu$ (the quantity $N$ is the ionic density). The ensemble-averaged magnetization reaches a peak value of $M=5.5 \times 10^{-3} \mathrm{~A} / \mathrm{m}$ and then sharply drops.

\section{VERIFYING THE MAGNETIC DIPOLE NATURE OF THE TRANSITION}

We now discuss how we verify that the electrons interact with the magnetic field of the laser during the ${ }^{7} F_{0} \rightarrow{ }^{5} D_{1}$ excitation. We note that we cannot use the method outlined in Ref. [35]. This is because our experiment uses a $\sim \mathrm{mm}$-scale crystal, and as a result, we cannot utilize the unique magnetic-field profile of a tightly focused radially polarized laser. Instead, we study angle-dependent fluorescence from the crystal with light propagating along each optical axis and for two orthogonal polarizations ( $S$ and $P$ polarized). This measurement is performed using a thicker crystal with dimensions $4 \times 5 \times 10 \mathrm{~mm}^{3}$ and outside the cryostat (i.e., at room temperature) so that we have access to all propagation axes of the crystal. The main idea behind this measurement is depicted in the schematic shown in Fig. 5. Consider $S$-polarized light propagating along a certain axis of the crystal, as shown in Fig. 5(a). The laser excites the crystal and we record the total fluorescence using a high-numerical-aperture lens and a photodetector (not shown in Fig. 5). If the excitation is due to interaction with the magnetic field, as the crystal is rotated along the

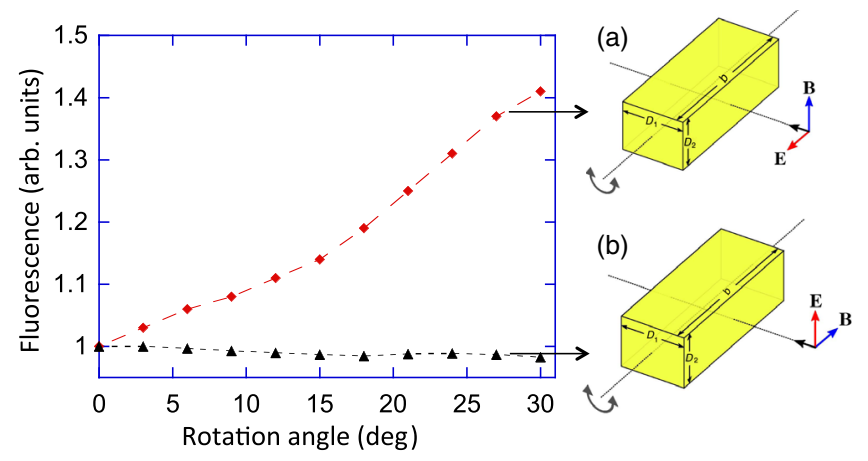

FIG. 5. Fluorescence from the crystal as a function of the angle of incidence for $S$-polarized [diamonds, schematic (a)] and $P$ polarized [triangles, schematic (b)] light. The fluorescence signal is normalized to take into account both the change in the Fresnel reflection losses from the surfaces and the path-length change of the beam as the crystal is rotated. The large increase in the fluorescence for $S$-polarized light (a) is due to a change of the direction of the angle of the magnetic field with the optical axes. In contrast, when the angle of the electric field is varied (b), there is negligible change in the fluorescence. This shows that during ${ }^{7} F_{0} \rightarrow{ }^{5} D_{1}$ excitation, light interacts with the magnetic field of light instead of the electric field. 
axis shown, we would expect a change in the amount of excitation (and, therefore, the amount of fluorescence recorded). This is because the crystal is highly anisotropic, and as the crystal is rotated, the angle between the magnetic field and the optical axes would change. For this geometry, we would not expect variation in the fluorescence if the excitation is due to the electric field since its orientation with respect to all axes remains unchanged. For $P$-polarized light [Fig. 5(b)], the roles of the electric and magnetic fields would be reversed. The plot in Fig. 5 shows this measurement for light propagating along the $D_{1}$ axis of the crystal with the crystal rotated around the $b$ axis. The measurement conclusively shows that the fluorescence changes when the direction of the $B$ field is varied, and therefore proves the magnetic-dipole nature of the excitation. We repeat this measurement for light propagating along the other axes; the rest of the data are presented in Appendix F.

\section{CONCLUSIONS AND FUTURE DIRECTIONS}

Here, we discuss several avenues of future work. Observing more Rabi cycles would allow the determination of the magnetic-dipole matrix element with better accuracy. This could be accomplished using, for example, spectral hole burning techniques [37,38]. Here, the idea is to select and optically pump a certain class of europium ions (whose transition frequencies lie within a certain frequency range) to one of the ground hyperfine levels (for example, to $\left| \pm \frac{1}{2}\right\rangle$ ). Rabi flopping can then be implemented using only this specific class of ions instead of the whole inhomogeneous profile.

Another direction would be the study of coherent and quantum interference effects in light-atom interactions. It is now well understood that electromagnetically induced transparency and related quantum interference effects can be observed in rare-earth ions using their hyperfine structure [42-44]. With the ions optically pumped to a specific hyperfine level, appropriately tuned probe and coupling laser beams can be used to form a $\Lambda$ scheme. For the first time, it will be possible to study these effects through the interaction of the electrons with the magnetic field of the laser beams.

A key application of this work is the recently suggested scheme of externally polarized and magnetized left-handed materials [29]. With the crystal magnetized, the electricdipole polarization can be induced a number of ways, for example, using higher-order nonlinearity of the host YSO crystal. Using this approach, the magnetization achieved in our experiment would allow the observation of left-handed electromagnetic waves with intensities of about $1 \mathrm{nW} / \mathrm{cm}^{2}$. As discussed in detail in Ref. [29], negative refraction of these waves from the crystal to air would also be observable. It is important to note that our experiment is far from achieving a negative permeability, which is the most direct route for constructing negative-index materials. Figure 6

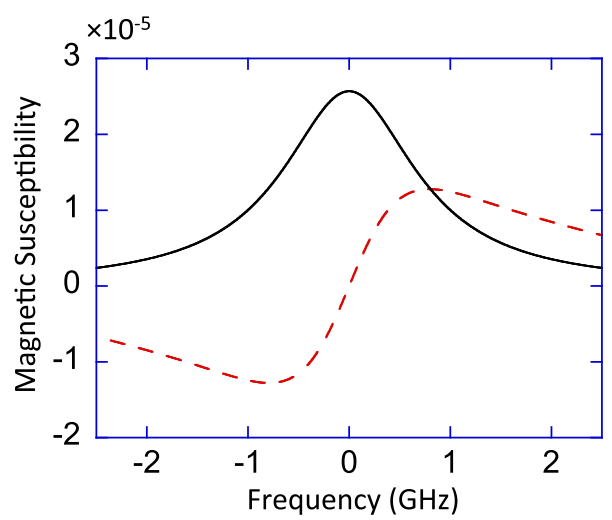

FIG. 6. The inferred real (dashed line) and imaginary (solid line) parts of the magnetic susceptibility as the laser frequency is scanned across the ${ }^{7} F_{0} \rightarrow{ }^{5} D_{1}$ transition. See Appendix $G$ for details.

shows the inferred real and imaginary parts of the magnetic susceptibility $\chi_{M}$ as the probe laser is scanned through the ${ }^{7} F_{0} \rightarrow{ }^{5} D_{1}$ transition (the permeability of the material is $\left.1+\chi_{M}\right)$. Both real and imaginary parts of the susceptibility exceed $10^{-5}$ near the resonance. Using stronger magneticdipole transitions and higher ionic densities, it will likely be possible to increase the magnetic susceptibility significantly. We also note that a negative permeability is not necessary to create a left-handed material. For example, using chirality, even a magnetic susceptibility of $\chi_{M} \approx 10^{-2}$ is sufficient to induce a negative index of refraction $[18,19]$.

\section{ACKNOWLEDGMENTS}

We would like to thank Dan Sikes for his important contributions during the early stages of this project. We also would like to thank David Gold, Dipto Das, Josh Karpel, and Ben Lemberger for many helpful discussions. This work has been supported by the Air Force Office of Scientific Research (AFOSR) and Wisconsin Alumni Research Foundation (WARF).

\section{APPENDIX A: EXPERIMENTAL SETUP}

The experiment starts with a custom-built external cavity diode laser at a wavelength of $1055 \mathrm{~nm}$, which uses a Littrow configuration for feedback. The ECDL produces an optical power of about $25 \mathrm{~mW}$ with an absolute linewidth of about $0.5 \mathrm{MHz}$. We lock the ECDL to an ultralow expansion glass cavity that serves as a frequency reference for the experiment. The ULE glass cavity is housed inside an ultrahigh vacuum chamber to reduce temperature fluctuations and mechanical perturbations to the cavity. The resonances of the ULE cavity have long-term frequency stability (over many days) better than $100 \mathrm{kHz}$. We use the Pound-Drever-Hall method to lock the frequency of the ECDL to one of the cavity resonances which is lined up with the peak of the inhomogeneous profile [45]. 
The ytterbium-doped fiber amplifier is commercial and amplifies the ECDL output to an optical power over $5 \mathrm{~W}$. We frequency double the amplifier output using cavity-based second harmonic generation (SHG). We use a 2-cm-long periodically poled KTP crystal for SHG. The poling period of KTP is adjusted for phase matching at $1055 \mathrm{~nm}$ and is temperature controlled with a temperature stability at the level of $1 \mathrm{mK}$. One of the mirrors of the SHG cavity is mounted on a piezoelectric transducer, which is used to lock the amplifier output to the SHG cavity using the HanschCouillaud method [46].

The acousto-optic modulators have a center frequency of $80 \mathrm{MHz}$ and a turn-on time of about $50 \mathrm{~ns}$. After the AOMs, both the probe and the Rabi laser beams are coupled to polarization-maintaining single-mode optical fibers. The fibers clean up the spatial profiles of the lasers and produce single-mode Gaussian beams which are then focused on the Eu:YSO crystal using lenses.

\section{APPENDIX B: CRYSTAL STRUCTURE AND STARK SPLITTINGS}

The YSO crystal is a monoclinic biaxial crystal of the $C_{2 h}^{6}$ space group. When doped, the $\mathrm{Eu}^{+3}$ ions can occupy two unique sites in the crystal, and these sites are typically referred to as "site 1" and "site 2." The transition frequencies differ by about $\sim 0.1 \mathrm{~nm}$ between the two sites. In our experiment, we use the $\mathrm{Eu}^{+3}$ ions of site 2 since the transition strengths are higher for this site.

In a free ion, the upper level ${ }^{5} D_{1}$ would have three degenerate levels $m_{J}=-1,0,1$. The crystal electric field breaks the degeneracy and splits the energy levels due to the Stark effect. The number of splittings depends on the symmetry properties of the crystal field. Because of the low symmetry of the crystal field in the YSO crystal, the $m_{J}$ levels of ${ }^{5} D_{1}$ are split into three. We use the Stark component with the lowest energy, $\left|J=1, m_{J}=0\right\rangle$, since it has the lowest phonon-induced nonradiative relaxation rate (and, therefore, the narrowest homogeneous linewidth).

\section{APPENDIX C: EXPERIMENTAL TIMING SEQUENCE}

With the ions initialized to their ground level, we apply a Rabi pulse of a certain duration to the ions. The duration of the Rabi pulse is adjusted by switching the AOM on and off. After the Rabi beam is turned off, we turn on a 5- $\mu s$ long probe pulse in a counterpropagation geometry and measure its transmission through the crystal. The probe power is attenuated to very low levels so that the ionic populations are not significantly perturbed due to the presence of the probe. The probe power that is transmitted through the crystal is measured with a commercial singlephoton counting module.

Because of the low operating temperature, the lifetimes of the ground hyperfine levels are quite long. We measure spectral hole lifetimes exceeding many minutes in our system. As a result, after each experiment, we need to reinitialize the ions to their ground hyperfine levels. We achieve this by slightly heating the crystal to a temperature of about $20 \mathrm{~K}$ and recooling it back to $4.5 \mathrm{~K}$ between two experimental pulse sequences.

\section{APPENDIX D: THEORETICAL CALCULATION OF THE MAGNETIC-DIPOLE MATRIX ELEMENT}

A detailed discussion for calculating transition matrix elements of lanthanides can be found in Ref. [41]. An important feature of the $4 f$ configuration in rare-earth metals is that the coupling is in the intermediate coupling regime. As a result, several interaction strengths in the problem are comparable, and neither $L S$ - nor $j j$-coupled basis states sufficiently approximate the real atomic states. The ionic states of our magnetic dipole transition are mixtures of states in the $L S$-coupled basis and they are given by $[39,41]$

$$
\begin{aligned}
\left|{ }^{7} F_{0}\right\rangle= & -0.968\left|{ }^{7} F_{0}\right\rangle+0.002\left|{ }^{5} D_{0}\right\rangle \\
& +0.166\left|{ }^{5} D_{0}^{\prime}\right\rangle-0.182\left|{ }^{5} D_{0}^{\prime \prime}\right\rangle, \\
\left|{ }^{5} D_{1}\right\rangle= & 0.210\left|{ }^{7} F_{1}\right\rangle-0.207\left|{ }^{5} D_{1}\right\rangle \\
& +0.716\left|{ }^{5} D_{1}^{\prime}\right\rangle-0.556\left|{ }^{5} D_{1}^{\prime \prime}\right\rangle,
\end{aligned}
$$

where' and " denote the seniority number. In the $L S$-coupled basis, the magnetic-dipole transitions obey the $\Delta L=0$ and $\Delta S=0$ selection rule. For magnetic-dipole transitions, the electric-dipole operator is replaced by the magnetic-dipole operator, which is given by

$$
\begin{aligned}
\mu^{(1)} & =-\mu_{B} \sum_{i}\left(l_{i}^{(1)}+g s_{i}^{(1)}\right) \\
& =-\mu_{B}\left(L^{(1)}+g S^{(1)}\right) \\
& =-\mu_{B}\left[J^{(1)}+(g-1) S^{(1)}\right] .
\end{aligned}
$$

Here, the $\sum_{i}$ summation is over all the relevant electrons, $L^{(1)}$ is the total orbital angular momentum, $S^{(1)}$ is the total spin angular momentum, and $J^{(1)}=L^{(1)}+S^{(1)}$. The superscript (1) refers to writing these vectorial quantities in the Racah tensor form. In Eq. (D2), the quantity $g$ is the anomalous gyromagnetic ratio for the electron spin $(g \approx 2)$. Using the pure $L S$-coupled basis functions, the reduced matrix element of the magnetic-dipole operator can be evaluated rather accurately [40]:

$$
\begin{aligned}
&\left\langle L S J\left\|J^{(1)}+S^{(1)}\right\| L^{\prime} S^{\prime} J^{\prime}\right\rangle \\
&=\delta_{L S, L^{\prime} S^{\prime}}[J(J+1)(2 J+1)]^{1 / 2} \\
&+\delta_{L S, L^{\prime} S^{\prime}}(-1)^{L+S+J+1}\left[(2 J+1)\left(2 J^{\prime}+1\right)\right]^{1 / 2} \\
& \times\left\{\begin{array}{ccc}
L & S & J \\
1 & J^{\prime} & S
\end{array}\right\}[S(S+1)(2 S+1)]^{1 / 2} .
\end{aligned}
$$


Here, $L, S$, and $J$ are the relevant quantum numbers of the ground level and $L^{\prime}, S^{\prime}$, and $J^{\prime}$ are the numbers of the excited level. The quantity $\left\{\begin{array}{ccc}L & S & J \\ 1 & J^{\prime} & S\end{array}\right\}$ is the Wigner $6 j$ coefficient. Using Eqs. (D2) and (D3), and the state mixtures of Eq. (D1), the reduced matrix element for our magnetic-dipole transition can be calculated to be

$$
\left\langle{ }^{7} F_{0}\left\|\mu^{(1)}\right\|^{5} D_{1}\right\rangle=0.16 \mu_{B} .
$$

With the reduced matrix element known, the matrix element for a specific polarization can be found using the Wigner-Eckart theorem. In our experiment, the magnetic field of the laser is along the quantization axis $(\pi$ polarization), which then gives $\left\langle{ }^{7} F_{0}, m_{J}=0\left|\mu_{0}^{(1)}\right|{ }^{5} D_{1}, m_{J^{\prime}}=0\right\rangle=$ $0.096 \mu_{B}$. Thus, the calculated value of the matrix element is $60 \%$ larger than our experimentally measured value. We think the likely reason for the discrepancy is the inaccurate values for the mixing coefficients of Eq. (D1).

\section{APPENDIX E: NUMERICAL SIMULATIONS}

In the numerical simulations, for each ion in the inhomogeneously broadened ensemble, we solve the time-dependent density-matrix equations using the fourthorder Runge-Kutta algorithm:

$$
i \hbar \frac{d \hat{\rho}}{d t}=[\hat{H}, \hat{\rho}]-i \frac{\hbar}{2}\{\hat{\Gamma}, \hat{\rho}\} .
$$

In the density matrix, we include all six hyperfine levels of the ${ }^{7} F_{0} \rightarrow{ }^{5} D_{1}$ transition as well as the ${ }^{5} D_{0}$ level. As a result, the size of the density matrix $\hat{\rho}$ is $7 \times 7$. The other ionic levels are sufficiently far detuned from the laser beams that they do not significantly affect the dynamics. Also, the ionic density is sufficiently low that ion-ion interactions are not of importance. At the start of the time evolution, each ion is equally probable to be in one of the hyperfine levels of the ground level ${ }^{7} F_{0}$; i.e., $\hat{\rho}_{11}(t=0)=\hat{\rho}_{22}(t=0)=$ $\hat{\rho}_{33}(t=0)=1 / 3$. The Hamiltonian operator $\hat{H}$ includes all laser couplings in the system due to magnetic-dipole interaction including all the relevant frequency detunings. The decay operator $\hat{\Gamma}$ includes nonradiative relaxation from the ${ }^{5} D_{1}$ level to ${ }^{5} D_{0}$, as well as the fluorescence lifetime of the ${ }^{5} D_{0}$ level. As we discuss above, we measure both of these decay rates using the fluorescence measurement of Fig. 2. Because we have an inhomogeneously broadened ensemble, to obtain accurate results, we perform the numerical integration for 80000 ions, whose resonance frequencies are shifted within the distribution of the inhomogeneous line shape. We include both isotopes of europium in our calculations. The ions in the wings of the inhomogeneous distribution have a large detuning from the Rabi laser beam since this laser is tuned to the center of the profile. As a result, numerical integration of the ions in the wings requires a significantly smaller time step size, which substantially increases the run time of the simulation. To alleviate this problem, we focus on the center of the inhomogeneous profile and consider only the ions whose frequencies lie within $1 \mathrm{GHz}$ of the line center. This is an excellent approximation since the ions in the wings contribute very little to the dynamics due to their large detuning from the laser. After numerical integration for each ion, we calculate the ensemble-averaged response by averaging the values of the density matrix elements at the end of numerical integration.

Europium nuclei have a nuclear spin of $I=5 / 2$, with corresponding spin projections $m_{I}$ ranging from $m_{I}=$ $-5 / 2$ to $m_{I}=5 / 2$. Nuclear states with different $m_{I}$ will have different energies due to a number of interactions. In europium, the dominant interactions are nuclear quadrupole and pseudoquadrupole interactions. A detailed discussion of these interactions can be found, for example, in Ref. [47]. For the ${ }^{153} \mathrm{Eu}^{+3}$ isotope in site 2, these interactions produce the hyperfine structure shown in Fig. 2. Because of its spin angular momentum, the nucleus will also have a magnetic moment. The total magnetic moment of the atom will be

$$
\mu^{(1)}=\mu_{B}\left(J^{(1)}+S^{(1)}\right)+\mu_{n} I^{(1)} .
$$

Here, $\mu_{n}$ is the nuclear magneton and its value is smaller than the Bohr magneton by the ratio of the electron mass to the proton mass. As a result, for pure nuclear spin states, the magnetic-dipole moment operator $\mu^{(1)}$ is diagonal:

$\left\langle m_{I}\left|\left\langle J m_{J}\left|\mu_{q}^{(1)}\right| J^{\prime} m_{J}^{\prime}\right\rangle\right| m_{I}^{\prime}\right\rangle=\delta_{m_{I} m_{I^{\prime}}}\left\langle J m_{J}\left|\mu_{q}^{(1)}\right| J^{\prime} m_{J}^{\prime}\right\rangle$.

Unfortunately, the situation is not as simple as described by the above equation. The reason is that there are a number of processes that mix the nuclear wave functions, and as a result, the nuclear spin states are mixed (see, for example, Ref. [48]). The hyperfine mixing coefficients for the ${ }^{5} D_{1}$ level are not known. These coefficients can be measured in the future by using spectral hole burning techniques. In our numerical simulations, we use the relative transition probabilities between the hyperfine levels of the ${ }^{7} F_{0} \rightarrow{ }^{5} D_{0}$ transition, as recently measured by Ref. [38]. Based on these transition probabilities, the dynamics are dominated by the three strongest transitions with Rabi frequencies of $0.92 \Omega, 0.86 \Omega$, and $0.82 \Omega$, respectively $(\Omega=B \mu / \hbar)$. We check that the numerical results are relatively insensitive to the exact values of the transition probabilities.

\section{APPENDIX F: ANGLE-DEPENDENT FLUORESCENCE MEASUREMENTS}

We perform the angle-dependent fluorescence measurements with light propagating along the other axes of the crystal. Similar to the measurements of Fig. 5, for light propagating along a specific crystal axis, we record the total fluorescence as the crystal is rotated for two polarizations of light. These measurements are shown in Fig. 7. For each propagation axis, the fluorescence changes when the direction of the magnetic field is changing, and stays relatively constant 

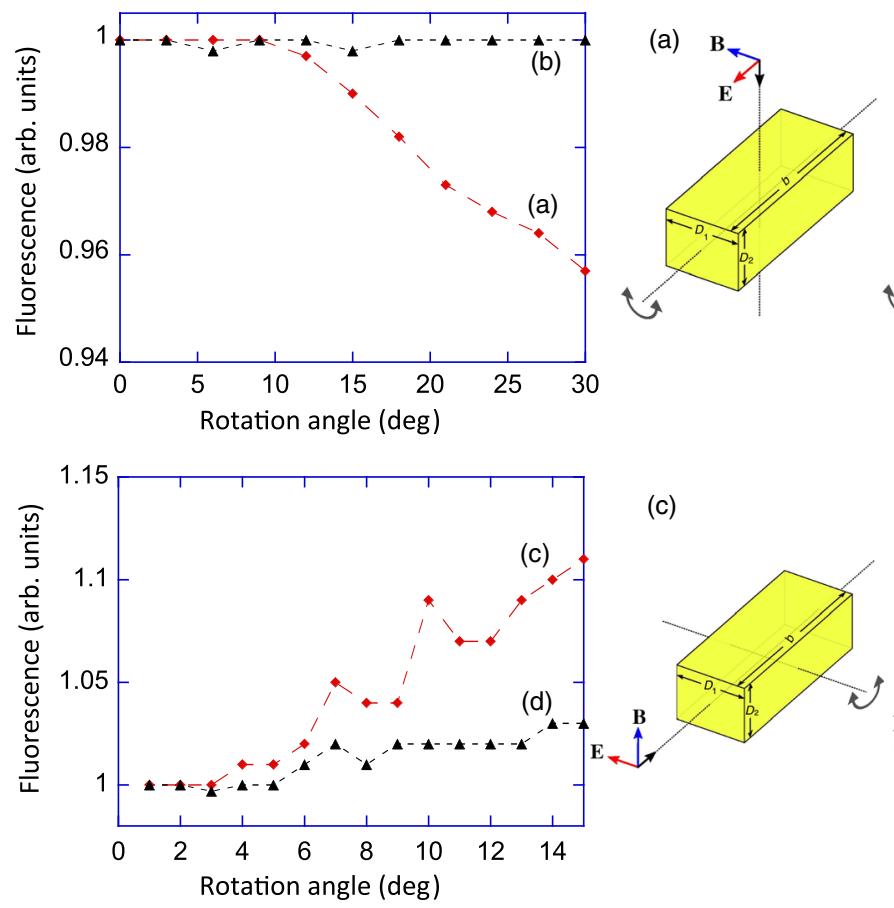

(b)

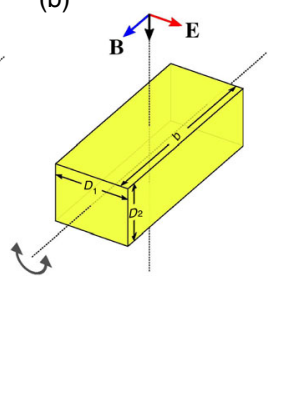

(d)

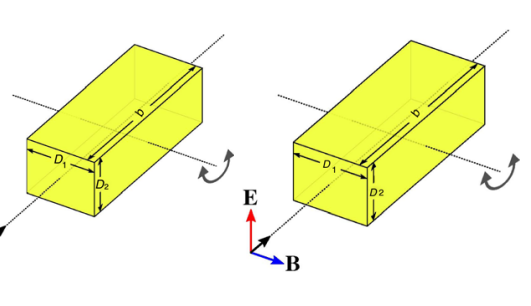

FIG. 7. Total fluorescence from the crystal as a function of the angle of incidence for light propagating along the $D_{2}$ axis (a), (b) and along the $b$ axis (c), (d) of the crystal. The measurements (c) and (d) suffer from low signal-to-noise ratio, but the overall trend is clear. For each propagation direction, the fluorescence depends sensitively on the direction of the magnetic field.

when the direction of the electric field is varied. The data points for Figs. 7(c) and 7(d) suffer from the technical limitations due to long propagation distance in the crystal.

\section{APPENDIX G: MAGNETIC SUSCEPTIBILITY}

For a certain class of ions, the magnetic susceptibility is given by

$$
\chi_{M}=N \mu_{0} \frac{\mu^{2}}{\hbar(\delta \omega-i \Gamma / 2)} .
$$

Here, $\mu_{0}$ is the permeability of free space and $\delta \omega$ is the frequency detuning of the probe laser beam from the magnetic-dipole transition. Equation. (G1) is the homogeneous line shape of the transition. We then convolve this line shape with the inhomogeneous profile to calculate the susceptibility for the whole ensemble (we note that inhomogeneous broadening produces a Lorentzian rather than a Gaussian distribution in these systems). The resulting convolved profile is displayed in Fig. 6.

[1] V. G. Veselago, The Electrodynamics of Substances with Simultaneously Negative Values of $\epsilon$ and $\mu$, Sov. Phys. Usp. 10, 509 (1968).

[2] J. B. Pendry, Negative Refraction Makes a Perfect Lens, Phys. Rev. Lett. 85, 3966 (2000).
[3] J. B. Pendry, A. Aubry, D. R. Smith, and S. A. Maier, Transformation Optics and Subwavelength Control of Light, Science 337, 549 (2012).

[4] R. A. Shelby, D. R. Smith, and S. Shultz, Experimental Verification of a Negative Index of Refraction, Science 292, 77 (2001).

[5] A. H. Houck, J. B. Brock, and I. L. Chuang, Experimental Observations of a Left-Handed Material that Obeys Snell's Law, Phys. Rev. Lett. 90, 137401 (2003).

[6] H. J. Lezec, J. A. Dionne, and H. A. Atwater, Negative Refraction at Visible Frequencies, Science 316, 430 (2007).

[7] N. Fang, H. Lee, C. Sun, and X. Zhang, Sub-DiffractionLimited Optical Imaging with a Silver Superlens, Science 308, 534 (2005).

[8] I. I. Smolyaninov, Y. Hung, and C. C. Davis, Magnifying Superlens in the Visible Frequency Range, Science 315, 1699 (2007).

[9] D. Schurig, J. J. Mock, B. J. Justice, S. A. Cummer, J. B. Pendry, A. F. Starr, and D. R. Smith, Metamaterial Electromagnetic Cloak at Microwave Frequencies, Science 314, 977 (2006).

[10] J. Valentine, J. Li, T. Zentgraf, G. Bartal, and X. Zhang, An Optical Cloak Made of Dielectrics, Nat. Mater. 8, 568 (2009).

[11] T. Ergin, N. Stenger, P. Brenner, J. B. Pendry, and M. Wegener, Three-Dimensional Invisibility Cloak at Optical Wavelengths, Science 328, 337 (2010).

[12] S. Linden, C. Enkrich, M. Wegener, J. Zhou, T. Koschny, and C. M. Soukoulis, Magnetic Response of Metamaterials at $100 \mathrm{THz}$, Science 306, 1351 (2004).

[13] S. Xiao, V. P. Drachev, A. V. Kildishev, N. Xingjie, U. K. Chettiar, Y. Hsiao-Kuan, and V. M. Shalaev, Loss-Free 
and Active Optical Negative-Index Metamaterials, Nature (London) 466, 735 (2010).

[14] A. D. Boardman, V. V. Grimalsky, Y. S. Kivshar, S. V. Koshevaya, M. Lapine, N. M. Litchinitser, V. N. Malnev, M. Noginov, Y. G. Rapoport, and V. M. Shalaev, Active and Tunable Metamaterials, Lasers Photonics Rev. 5, 287 (2011).

[15] A. A. High, R. C. Devlin, A. Dibos, M. Polking, D. S. Wild, J. Perczel, N. P. de Leon, M. D. Lukin, and H. Park, VisibleFrequency Hyperbolic Metasurface, Nature (London) 522, 192 (2015).

[16] M. O. Oktel and O. E. Mustecaplioglu, Electromagnetically Induced Left-Handedness in a Dense Gas of Three-Level Atoms, Phys. Rev. A 70, 053806 (2004).

[17] Q. Thommen and P. Mandel, Electromagnetically Induced Left Handedness in Optically Excited Four-Level Atomic Media, Phys. Rev. Lett. 96, 053601 (2006).

[18] J. Kästel, M. Fleischhauer, S. F. Yelin, and R. L. Walsworth, Tunable Negative Refraction without Absorption via Electromagnetically Induced Chirality, Phys. Rev. Lett. 99, 073602 (2007).

[19] D. E. Sikes and D. D. Yavuz, Negative Refraction with Low Absorption Using Raman Transitions with Magneto-Electric Coupling, Phys. Rev. A 82, 011806(R) (2010).

[20] P. P. Orth, R. Hennig, C. H. Keitel, and J. Evers, Negative Refraction with Tunable Absorption in an Active Dense Gas of Atoms, New J. Phys. 15, 013027 (2013).

[21] M. O. Scully and M.S. Zubairy, Quantum Optics (Cambridge University Press, Cambridge, England, 1997).

[22] S. E. Harris, Electromagnetically Induced Transparency, Phys. Today 50, 36 (1997).

[23] O. Kocharovskaya and P. Mandel, Amplification without Inversion, The Double- $\Lambda$ Scheme, Phys. Rev. A 42, 523 (1990).

[24] L. V. Hau, S. E. Harris, Z. Dutton, and C. H. Behroozi, Light Speed Reduction to $17 \mathrm{~m} / \mathrm{s}$ in an Ultracold Atomic Gas, Nature (London) 397, 594 (1999).

[25] M. Fleischhauer and M. D. Lukin, Dark State Polaritons in Electromagnetically Induced Transparency, Phys. Rev. Lett. 84, 5094 (2000).

[26] G. M. Gehring, A. Schweinsberg, C. Barsi, N. Kostinski, and R. W. Boyd, Observation of Backward Pulse Propagation through a Medium with a Negative Group Velocity, Science 312, 895 (2006).

[27] N. B. Phillips, A. V. Gorshkov, and I. Novikova, Optimal Light Storage in Atomic Vapor, Phys. Rev. A 78, 023801 (2008).

[28] M. D. Lukin and A. Imamoglu, Nonlinear Optics and Quantum Entanglement of Ultraslow Photons, Phys. Rev. Lett. 84, 1419 (2000).

[29] D. D. Yavuz and N. R. Brewer, Left-Handed Electromagnetic Waves in Materials with Induced Polarization and Magnetization, Phys. Rev. A, 90, 063807 (2014).

[30] G. H. Dieke and H. M. Crosswhite, The Spectra of the Doubly and Triply Ionized Rare Earths, Appl. Opt. 2, 675 (1963).
[31] B. G. Wybourne, Spectroscopic Properties of Rare-Earths (John Wiley \& Sons, New York, 1965).

[32] R. M. Macfarlane, High-Resolution Laser Spectroscopy of Rare-Earth Doped Insulators: A Personal Perspective, J. Lumin. 100, 1 (2002).

[33] M. J. Weber and R. F. Schaufele, Radiative and Nonradiative Transitions in the Fluorescence Decay of $\mathrm{Eu}^{+3}$ in $\mathrm{SrTiO}_{3}$, J. Chem. Phys. 43, 1702 (1965).

[34] W. F. Krupke, Optical Absorption and Fluorescence Intensities in Several Rare-Earth-Doped $\mathrm{Y}_{2} \mathrm{O}_{3}$ and $\mathrm{LaF}_{3}$ Single Crystals, Phys. Rev. 145, 325 (1966).

[35] M. Kasperczyk, S. Person, D. Ananias, L. D. Carlos, and L. Novotny, Excitation of Magnetic Dipole Transitions at Optical Frequencies, Phys. Rev. Lett. 114, 163903 (2015).

[36] X. A. Shen and R. Kachru, ${ }^{7} F_{0} \rightarrow{ }^{5} D_{1}$ Transition in Eu: YSO, J. Opt. Soc. Am. B 11, 591 (1994).

[37] F. Konz, Y. Sun, C. W. Thiel, R. L. Cone, R. W. Equall, R. L. Hutcheson, and R. M. Macfarlane, Temperature and Concentration Dependence of Optical Dephasing, Spectral-Hole Lifetime, and Anisotropic Absorption in $\mathrm{Eu}^{+3}$ : $\mathrm{Y}_{2} \mathrm{SiO}_{5}$, Phys. Rev. B 68, 085109 (2003).

[38] B. Lauritzen, N. Timoney, N. Gisin, M. Afzelius, H. de Rietmatten, Y. Sun, R. M. Macfarlane, and R. L. Cone, Spectroscopic Investigations of $\mathrm{Eu}^{+3}: \mathrm{Y}_{2} \mathrm{SiO}_{5}$ for Quantum Memory Applications, Phys. Rev. B 85, 115111 (2012).

[39] G. S. Ofelt, Structure of the $f^{6}$ Configuration with Application to Rare-Earth Ions, J. Chem. Phys. 38, 2171 (1963).

[40] R. D. Cowan, The Theory of Atomic Structure and Spectra (University of California Press, Berkeley, 1981).

[41] B. G. Wybourne and L. Smentek, Optical Spectroscopy of Lanthanides (Taylor \& Francis, London, 2007).

[42] B. S. Ham, P. R. Hemmer, and M. S. Shahriar, Efficient Electromagnetically Induced Transparency in a Rare-Earth Doped Crystal, Opt. Commun. 144, 227 (1997).

[43] A. V. Turukhin, V. S. Sudarshanam, M. S. Shahriar, J. A. Musser, B.S. Ham, and P. R. Hemmer, Observation of Ultraslow and Stored Light Pulses in a Solid, Phys. Rev. Lett. 88, 023602 (2001).

[44] J. Klein, F. Beil, and T. Halfmann, Experimental Investigations of Stimulated Raman Adiabatic Passage in a Doped Solid, Phys. Rev. A 78, 033416 (2008).

[45] R. W. P. Drever, J. L. Hall, F. V. Kowalski, J. Hough, G. M. Ford, A. J. Munley, and H. Ward, Laser Phase and Frequency Stabilization Using an Optical Resonator, Appl. Phys. B 31, 97 (1983).

[46] T. W. Hansch and B. Couillaud, Laser Frequency Stabilization by Polarization Spectroscopy of a Reflecting Reference Cavity, Opt. Commun. 35, 441 (1980).

[47] J. J. Longdell, A. L. Alexander, and M. J. Sellars, Characterization of the Hyperfine Interaction in Europium-Doped Yttrium Orthosilicate and Europium Chloride Hexahydrate, Phys. Rev. B 74, 195101 (2006).

[48] M. Nilsson, L. Rippe, S. Kroll, R. Klieber, and D. Suter, Hole-Burning Techniques for Isolation and Study of Individual Hyperfine Transitions in Inhomogeneously Broadened Solids Demonstrated in Pr:YSO, Phys. Rev. B 70, 214116 (2004). 